\title{
Las normas jurídicas: una aproximación desde el convencionalismo jurídico y el análisis económico del derecho *
}

\section{Legal rules: an approach from the Legal Conventionalism and Economic Analysis of Law}

\author{
${ }^{*}$ Luis Fernando Bermeo Álvarez
}

\begin{abstract}
** Estudiante de Maestría en Derecho con énfasis en investigación de la Universidad del Norte. Docente universitario en la Fundación Tecnológica Comfenalco. Investigador del grupo de investigación "Filosofía del Derecho y Derecho Constitucional". Correos: luisfernandobermeoalvarez@gmail.com - lbermeo@uninorte.edu.co.Colombia.
\end{abstract}

Cómo citar: Bermeo, L.F.(2016). Las normas jurídicas: una aproximación desde el convencionalismo jurídico y el análisis económico del derecho Inciso 18 (1) 99-107.

http://dx.doi.org/10.18634/incj.18v.1i.563

Recibido:03/02/2016 Revisado: 04/04/2016 Aceptado: 17/06/2016

\section{Resumen}

La Teoría General del Derecho ha venido cambiando a lo largo del desarrollo de la Globalización como fenómeno económico y cultural. Desde esta perspectiva el estudio del Derecho también ha ido sufriendo modificaciones tendientes a explorar nuevas formas de compresión y promoción del fenómeno jurídico. En este trabajo se ofrecen dos análisis sobre la forma de concebir el derecho en una sociedad: primero, desde una perspectiva descriptiva, que obedece al convencionalismo jurídico, en la que se presentan algunos resultados de investigación sobre el concepto de existencia de las normas jurídicas, en los que se trata de explicar dos aspectos claves en la creación del derecho, uno social (convencionalismo) y otro sistémico (positivismo); y segundo, desde una perspectiva funcional, en la que se exponen los resultados de una investigación preliminar sobre la operancia de las normas jurídicas al interior de una sociedad a partir de la Teoría del Análisis Económico del Derecho, en la que el papel de las normas jurídicas es visto como precio. Al final se sintetizan algunas conclusiones sobre los puntos comunes de estas dos aproximaciones.

Palabras clave: Análisis económico del derecho, convencionalismo jurídico, Estado de derecho, globalización, norma jurídica.

\begin{abstract}
The General Theory of Law has been changing along the development of globalization as an economic and cultural phenomenon. From this perspective the study of law has also been going through changes, aimed to explore new ways of compression and promotion of the legal phenomenon. This study offers two analysis of the way of conceiving the law of a society, first, from a descriptive perspective, which responds to the legal conventionalism, in which the research results will be presented on the concept of existence of legal rules, that tries to explain two key aspects in the creation of law, one social (conventionalism) and other systemic (positivism); and second, from a functional perspective, in which will be presented the preliminary results of an investigation about the operation of legal norms within a society using the theory of Economic Analysis of Law, where the role of legal rules is seen as price. At the end it will be synthesized some conclusions about the common points between these two approaches.
\end{abstract}

Keywords: Globalization, Legal Conventionalism, Economic Analysis of Law, Rule of Law, Legal Rules.

\footnotetext{
* Los resultados presentados en este trabajo sobre el Convencionalismo Jurídico son producto del proyecto de investigación "La eficacia simbólica del derecho a la Paz en Colombia" realizado en la Universidad del Norte, con la dirección del Dr. Juan Pablo Isaza Gutiérrez.
} 


\section{Introducción}

El fenómeno social denominado globalización ha traído consigo un replanteamiento de los sistemas jurídicos en casi todo el mundo (Teubner, 2010). La afectación de este fenómeno al campo jurídico resulta evidente en el contexto de proliferación de formas de resolver los conflictos por parte de los ciudadanos de todo el mundo, sin recurrir a fórmulas tradicionales, en este sentido, indica Teubner que "en todo el mundo crece la propagación infecciosa de la regulación privada, los acuerdos privados y la resolución privada de conflictos" (2010: 71).

A pesar de que este autor no es partidario de una explicación eminentemente económica, sino más bien cultural, holística y compleja, es posible reconocer que la globalización coloca en una posición de superioridad determinante socialmente a los regímenes privados frente a los públicos, dado que son los primeros los legítimamente autorizados para preocuparse y buscar únicamente su beneficio propio, sin necesidad de preguntarse, aunque sea hipócritamente, por intereses altruistas o que beneficien a un mayor número de personas, esto es problema del Estado (Mercado, 2008: 224).

Así las cosas, la globalización tiene profundas raíces determinantes en la economía, lo cual no implica que no existan consecuencias que se sigan de este fenómeno hacia otros ámbitos sociales como el derecho (Teubner, 2010:67 - 69).

Uno de los resultados de este fenómeno es la evidente importación del modelo de sistema jurídico anglosajón a la mayor parte del mundo. Esta observación podría parecer a primera vista propia de teorías conspiracionistas, sin embargo, está más que fundada.

Cesar Rodríguez (2008) planteó esta cuestión en $L a$ globalización del Estado de derecho y evidenció la intención de muchas instituciones mundiales, tanto estados como organismos no gubernamentales, empresas, etc., de imponer un solo modelo de Estado de derecho que permitiera hacer más eficiente las relaciones sociales (con fundamento económico) en el marco de la globalización.
Rodríguez (2008) ubica este fenómeno de estandarización del Estado de derecho a partir de la década de los años 60 y 70 en Estados Unidos, y posteriormente en los años 90 en este mismo país. El énfasis de este proceso se encuentra en el sistema judicial, es decir, en el sistema de acceso a la justicia y asignación de derechos por parte de los jueces con miras a la resolución de los conflictos, por lo que el sistema judicial ha de reformarse, atendiendo a principios económicos de eficiencia como agilidad y fácil acceso.

No en vano muchas teorías respecto al derecho que se enseñan desde hace tiempo en las facultades colombianas responden a paradigmas anglosajones, un ejemplo de ello es la famosa obra de H.L.A Hart (1963) El concepto de derecho, que está pensada para explicar el contexto histórico y cultural del autor, no todos los ordenamientos jurídicos del mundo, sin embargo, han sido semilla de importantes debates académicos e incluso, decisiones judiciales. Más allá de la teoría, también los regímenes procesales y sustanciales de los países han ido tendiendo a reformas cada vez más anglosajonas, bajo el paradigma de la oralidad, ejemplo de ello el Código general del proceso y la Ley 906 en Colombia. Otra ilustración sería la relevancia de los tribunales de arbitramento y centro de conciliación para dirimir conflictos en especial de órdenes económicos, muy propios de países con fuerte economía.

Desde esta perspectiva se puede estar de acuerdo con Teubner cuando dice que "la globalización produce un conjunto de problemas intrínsecos del derecho, relacionados con el cambio en los procesos dominantes de creación de normas" (Teubner, 2010: 69).

Teniendo en cuenta lo anterior, en este trabajo se presentarán los resultados de dos investigaciones exploratorias que fueron realizadas de manera independiente, pero que arrojaron aproximaciones similares hacia un mismo problema, que pueden resultar complementarias a la hora de explicar i) el origen y ii) la función de las normas jurídicas, en el contexto de la globalización del Estado de derecho. Ambos resultados se encuentran ubicados dentro del ámbito de la Teoría General del Derecho en el marco del fenómeno de la globalización. El primer 
análisis que se presentará será la aproximación al concepto de existencia de las normas jurídicas desde el convencionalismo jurídico, y el segundo será sobre la concepción de las normas jurídicas desde la teoría del Análisis Económico del Derecho.

Se considera que la primera visión sirve para describir el proceso de surgimiento y mantenimiento de las normas jurídicas en una sociedad, mientras que la segunda prescribe una forma de concebir las normas jurídicas desde presupuestos epistemológicos tendientes a una concepción del derecho más eficiente, que ayude a resolver los problemas sociales y jurídicos de mejor forma.

\section{Sobre el convencionalismo jurídico}

Tomando la regla de reconocimiento como criterio fundamental de existencia del sistema jurídico, se desprende una concepción del Derecho en la que lo jurídico está formado en su esencia por un tipo de experiencias sociales que se han denominado jurídicas al interior de una sociedad. Una de esas formas es el contacto del individuo con las normas y otra, la vivencia de diferentes hechos sociales por parte de individuo.

En este análisis preliminar la cuestión de fondo sobre la forma de concebir el objeto del Derecho consiste en: i) establecer si son las normas (normativismo) o los hechos sociales (convencionalismo), los conceptos relevantes para describir el criterio de existencia del Derecho, tanto para su estudio como para su práctica y ii) si es correcta o no, la presunta antítesis entre ambos elementos para describir el fenómeno jurídico. En principio, así ha sido presentada la relación entre normas jurídicas y hechos relativos al Derecho, sin embargo, pueden darse relaciones de conjunción entre ambas formas de expresión de lo jurídico.

En todo caso, es importante tener presente una doble dimensión del concepto de normas jurídicas, en tanto son concebidas como expresiones del lenguaje (lingüística), y como la realización de conductas en el seno de una sociedad (sociología).

Una de las corrientes iusfilosóficas que se ha dedicado a estudiar el problema del surgimiento del Derecho o de la esencia del Derecho ha sido el convencionalismo jurídico, del cual se puede decir de una forma preliminar que centra su análisis en la premisa fundamental de que "un determinado hecho jurídico es (determinado por) una convención" (Arena, 2012: 279).

Según esta teoría, "el convencionalismo consiste en una doble propuesta, por un lado un concepto de convención, por otro lado, la individualización de un ámbito del fenómeno jurídico al cual aplicar ese concepto" (Arena, 2012:278). A su vez, es posible identificar una relación teórica entre el convencionalismo jurídico y el positivismo jurídico, tal como lo formulan Papayannis y Ramírez (2012: 203): "el positivismo concibe el Derecho como una práctica social convencional". El punto de inicio que la teoría del Derecho ha identificado para el surgimiento del convencionalismo jurídico ha sido la teoría hartiana del Derecho (Arena, 2012: 287). Sin embargo, es posible ir más atrás en el tiempo y encontrar en la sociología una explicación convencionalista del Derecho a partir de Max Weber.

En su gran obra Wirtschaft und Gessellschat. Grundiss ver Verstehenden Soziologie (1922) publicada después de su muerte, Max Weber conceptuó el Derecho como un orden legítimo que "está garantizado externamente por la probabilidad de la coacción (física o psíquica) ejercida por un cuadro de individuos instituidos con la misión de obligar a la observancia de ese orden o castigar su trasgresión" (Weber, 2012: 27).

La definición anterior no se aleja del rasgo fundamental del Derecho como autoridad, contribución atribuida a prominentes teóricos del Derecho de tradición positivista, sin embargo, lo importante aquí es hacer hincapié en la relación entre Derecho y convención que distingue Weber en su clasificación de ordenes legítimos. Para él, un orden legítimo es una representación de la acción social por parte de sus partícipes, y la legitimidad de este orden puede ser garantizada internamente o "por la expectativa de determinadas consecuencias externas; o sea, por una situación de intereses" (Weber, 2012, 27). En este contexto, "un orden debe llamarse: a) convención o b) Derecho". Ahora bien, para Weber (2012):

Un orden debe llamarse convención: cuando su validez está garantizada externamente por la 
probabilidad de que, dentro de un determinado circulo de hombres, una conducta discordante habrá de tropezar con una (relativa) reprobación general y prácticamente sensible (Weber, 2012: 27).

Bajo esta conceptualización es posible identificar de manera general las convenciones con las reglas sociales, de tal forma que se pueden incluir los usos y/o la costumbre dentro de las reglas sociales o convenciones en el marco de la teoría weberiana. Sin embargo, hay algo más que decir sobre la relación entre Derecho y convención para Weber. Tal como se ha señalado, ambos conceptos incluyen el reproche social como punto en común, solo que mientras que para que exista Derecho se necesita de "un cuadro de individuos instituidos con la misión de obligar a la observancia de ese orden o castigar su trasgresión" (pág. 27), para la convención no se necesita este cuadro de individuos. Es decir, que el concepto de Derecho está fuertemente ligado al concepto de Estado, mientras que el concepto de convención se encuentra en el núcleo de toda organización social. Por lo tanto, no puede haber sociedad sin convención, ni Estado (moderno) sin Derecho.

Por otra parte, todo Estado es una organización social, por lo que en el fondo, es probable que exista en él una regla social nuclear que lo fundamente. En este sentido, el mito fundacional del contrato social no es otra cosa que una convención. En la misma noción de Estado (moderno), la primera convención es la limitación de ciertas libertades "naturales", para poder disfrutar de libertades "civiles" (Mercado, 2008: 38 - 39, 116 - 117). La cesión de libertades, o el contrato, se hace del individuo hacia Estado, quien "debe proteger esos Derechos y tiene el deber jurídico de hacerlos respetar” (pág. 39, 117 - 118).

Teniendo en cuenta lo dicho, resulta pertinente reproducir una cita de Max Weber (2012) para dejar sentada la diferencia fundamental entre convención y Derecho:

Lo que falta únicamente es el cuerpo de personas especialmente destinado a mantener su cumplimiento (juez, fiscales, funcionarios administrativos, etc.). Sin embargo, la transición es fluida. El caso límite de la garantía convencional de un orden, ya en tránsito hacia la garantía jurídica, se encuentra en la aplicación del boycot formalmente organizado y proclamado (en su amenaza). Esto, en nuestra terminología sería ya un medio de coacción jurídica (pág. 28).

Ahora bien, para Vilajosana (2010) una convención es un hecho social (pág. 154) o una práctica social (pág. 161), que puede estar vinculada a una regla social siempre que exista un criterio interno de obligatoriedad (recurriendo al denominado punto de vista interno de la regla de reconocimiento en la teoría de Hart) (pág. 161 - 162). Desde esta perspectiva, las normas jurídicas surgen de la convención que una sociedad determina para el sistema jurídico (o Derecho de esta sociedad) que será obligatorio al interior de esta sociedad.

Lo anterior no significa que exista un acuerdo al interior de la sociedad entre los individuos, tal como lo postula el mito fundacional del contractualismo, sino que esta convención surge como una solución práctica a problemas de coordinación recurrentes entre los individuos (pág. 162). El aspecto normativo de la convención viene dado de una parte por la solución práctica al problema de coordinación y del criterio interno de obligatoriedad sobre esta convención. En este punto merece ser expuesto el concepto de problema de coordinación siguiendo las precisiones de Federico Arena (2014):

Hay situaciones en las que dos o más individuos se enfrentan a más de una posibilidad de acción, algunas de las cuales satisfacen igualmente el interés de cada uno de ellos, con la condición de que cada uno lleve a cabo la misma acción (Pág. 100).

Vale decir que para Arena la solución a los problemas de coordinación es a su vez una convención de coordinación, en contraste con las convenciones de constitutivas, sobre las que no se ahondará en este trabajo. Un ejemplo de problemas de coordinación entre dos personas fáciles de identificar es el de dos individuos que mantienen una conversación telefónica y durante ella, en un momento determinado, se corta la comunicación, frente a lo cual, en caso de querer seguir hablando, tendrán que decidir cada uno por su cuenta qué hacer para volver a la conversación.

Cada uno tiene algunas de las siguientes posibilidades: a) volver a llamar y b) no volver a llamar. Si ambos 
optan al mismo tiempo por a), es posible que no logren coordinarse y por ende, no se restablezca la comunicación. Si ambos optan por b), es seguro que no se restablecerá la comunicación, por lo que, teniendo en cuenta que ambos quieren seguir hablando, uno tendrá que no llamar (esperar), mientras el otro tendrá que llamar para que puedan seguir comunicándose (Arena, 2014: 102).

Las consecuencias de las decisiones que tomen los individuos del ejemplo anterior son probables que determinen los resultados de futuros problemas de coordinación del mismo tipo. Una convención que podría surgir es que uno de los individuos siempre llame al otro. El hecho que se concrete la regularidad de que uno de los dos siempre llame y el otro siempre espere, desencadena necesariamente una convención de coordinación.

Una solución (quizás) definitiva a este problema sería establecer un acuerdo entre ambos individuos, por ejemplo, que A siempre llame a B cuando ocurra $\mathrm{C}$ (perdida de comunicación telefónica), lo que generaría una norma entre ambos.

Como este ejemplo puede darse otro a mayor clase social, es decir, entre un mayor número de individuos que componen una sociedad. Ejemplos de este tipo de problemas abundan, como la determinación del sentido de conducción vehicular en una carretera (pág. 102), la viabilidad de obras de infraestructura en una ciudad, hasta la determinación de una elección popular o una medida normativa, siempre y cuando se comparta un interés común.

Lo anterior no implica que siempre una convención esté determinada por un acuerdo (Arena, 2014: 80-82; Arena, 2012: 280). En primera medida "la existencia de una convención depende de un comportamiento de los involucrados" (Arena, 2012: 282). Para ilustrar mejor este punto vale la pena traer a colación la explicación del propio Federico Arena (2012):

En este sentido, una primera condición para la existencia de una convención sin acuerdo es que se verifique una cierta regularidad o convergencia de comportamiento. Pero, ¿en qué consiste esta regularidad? Una regularidad de comportamiento puede ser concebida como $<<$ realizar la acción A en la ocasión $\mathrm{O}>>$, o puesto de modo más general
$<<$ A en $\mathrm{O}>>$ (Gilbert, 2008, pág. 8). La regularidad exige que $\mathrm{O}$ se presente con cierta frecuencia, o al menos que se haya presentado más de una vez y que sea posible que vuelva a presentarse (Gilbert, 1983, pág. 229). Así, existe una regularidad de comportamiento en un grupo G, cuando los miembros de $\mathrm{G}$ realizan la acción A cada vez que se presenta la ocasión O (pág. 282).

Siguiendo este análisis, la segunda condición que se presenta para la existencia de una convención sin acuerdo es la "condición de dependencia" (pág. 283). En este sentido, "la condición de dependencia se refiere a las razones que llevan a la existencia de una convención" (pág. 283). Estas razones pueden ser muy variadas, pero en principio, una visión simple sería la que implica la regularidad de comportamiento de individuos de un grupo en determinada ocasión por el hecho que la mayoría "o los demás también lo hacen” (pág. 284). Para Arena (2012):

Bajo una versión más compleja, la condición de dependencia se verifica, respecto de una regularidad de comportamiento, si la razón para conformarse a la regularidad es, en parte, i. que los demás lo hacen y ii. que lo hacen por esta razón. En esta segunda versión, la dependencia es recíproca o refleja (pág. 284).

Por último, el tercer requisito para la existencia de una convención sin acuerdo es la arbitrariedad (pág. 285). En el núcleo central del concepto de arbitrariedad se encuentra la alternativa, es decir, "la existencia de un modo distinto de configurar una determinada convención" (pág. 285), en este punto Arena (2012) presenta dos convenciones triviales que no dependen de un acuerdo al interior de la sociedad (pág. 285):

a) Que los padres de la novia sufraguen los gastos de la fiesta de matrimonio. Es claro que el autor no se encuentra en posición de determinar si esta convención es válida o no en Colombia, o más aún en el Caribe colombiano, sin embargo, demos por sentado este ejemplo aunque sea de forma hipotética. Lo arbitrario del ejemplo consiste en que podría existir una opción distinta a que el pago de estos gastos corra por cuenta de la familia de la novia; bien podrían ser los padrinos de bodas, la familia del novio, los amigos de la pareja, o la pareja misma, etc. 
b) El sentido de circulación vehicular. En Colombia por ejemplo se conduce por la derecha y el timón de los carros también se encuentra ubicado en este lado del vehículo, a diferencia del Reino Unido donde es precisamente lo contrario; en esto consiste la alternativa.

¿Hubo algo así como un referendo para determinar estas convenciones? ¿Se firmó acaso un contrato social que definiría el sentido de conducción vehicular y quien iba a ser el encargado de sufragar los gastos de las fiestas matrimoniales entre las parejas?

Lo anterior prueba, aunque sea de manera hipotética e intuitiva, que estos ejemplos valen para demostrar la existencia de convenciones sin acuerdos. En definitiva, para que estos existan deben darse: "la convergencia de comportamiento, la condición de dependencia y la arbitrariedad" (Arena, 2012: 286). Una convención (sin acuerdo) importante para la creación del Derecho en una sociedad es la convergencia de comportamientos en torno a la idea de lo Justum, en este sentido para Viehweg (1997):

Toda agrupación social de personas que aspira a perdurar en el tiempo, a fin de posibilitar su cohesión, tiene que crear y fijar una opinión acerca de lo que considera como justum. Una opinión jurídica así fijada - cualquiera que fuese su fundamentación - tiene que ser entendida como una respuesta vinculante a una problemática social (Legaz y Lacambra, 1953, pág. 177) y cumple una función social al posibilitar el comportamiento de una pluralidad de personas (págs. 15-16)

Es esta idea compartida la que una vez fijada en determinada sociedad, empieza a imperar de forma obligatoria, a la vez que funda una asociación política (Weber, 2012: 43 - 44), conformada por individuos revestidos de autoridad encargados de mantener "la pretensión al monopolio legítimo de la coacción física para el mantenimiento del orden vigente" (Pág. 44), configurándose el fenómeno social del Derecho.

En este contexto el Derecho podría ser descrito en principio como un conjunto de experiencias compartidas por los miembros de una sociedad, los cuales han hecho surgir las normas con carácter jurídico a partir de sus convenciones y han otorgado obligatoriedad a esas normas a partir la escogencia de un grupo de individuos encargados de asegurarlas. También podría ser descrito en un segundo momento, como un conjunto de normas jurídicas aplicadas por ciertos individuos revestidos de autoridad legítima dentro de una determinada sociedad, organizada con arreglo al cumplimiento de dichas normas jurídicas.

\section{Sobre el análisis económico del Derecho (la visión de las normas jurídicas)}

El análisis económico del derecho parte de una visión del hombre bajo el "supuesto general de un sujeto maximizador que, en un contexto de escasez, busca maximizar su utilidad manteniendo estables sus preferencias" (Cossío, 2008: 237).

En este sentido, la premisa fundamental para el estudio del Derecho desde esta óptica es la utilización de un método propio de la economía para estudiar el Derecho, en especial de las teorías de la economía neoclásica, por ende, el análisis económico del derecho no es "sino un método para analizar aquellas conductas humanas que se encuentran relacionadas, de algún modo, con el derecho" (Cossío, 2008: 228). Para Cossío (2008):

De este modo y siguiendo a Becker, cabe decir que lo determinante para llegar a considera la "existencia" de una conducta económica es la suposición de que quien la realiza busca dentro de un contexto de escasez y manteniendo estables sus preferencias, "maximizar su utilidad. (pág. 197).

Teniendo en cuenta lo anterior, "para precisar el objeto del análisis económico del derecho, diremos que se trata del estudio de conductas económicas realizadas en condiciones determinadas por normas jurídicas" (Cossío, 2008: 238). Por otra parte, "la función de las normas jurídicas en el análisis económico del derecho es posibilitar la determinación -insistimosde las condiciones o ámbitos en que habrá de llevarse a cabo la maximización" (Cossío, 230 - 231). En este contexto el análisis económico del derecho ve las normas jurídicas como precios (Morales, 2011: 59) y los precios como costos de oportunidad, y el sistema jurídico como al mercado, a partir del supuesto antropológico (al que ya hemos hecho referencia) del hombre como ser racional y maximizador de sus 
beneficios. Las normas jurídicas se utilizan "como sistemas de incentivos para sus destinatarios y en los cálculos racionales de los sujetos se les asigna un precio" (Morales, 2011: 59).

Según el análisis económico del derecho las normas funcionan como sistemas de incentivos para sus destinatarios y en los cálculos racionales de los sujetos se les asigna un precio (Morales, 2011: 59). Para Pérez (2014):

La teoría del desincentivo afirma que las personas son seres racionales que evalúan costos y beneficios y que actúan conforma a los cacusos que hacen de estos. El comportamiento humano, según la teoría, es determinado por los premios y castigos existentes (y conocidos), así como por la percepción de ser castigado o premiado. Las personas, hacen un cálculo sobre las probabilidades de sanción y modulan su comportamiento en función de ello (pág. 16).

El sistema de precios identificado como normas jurídicas según el análisis económico del derecho funciona incentivando o desincentivando conductas al interior de la sociedad, en este sentido existirán costos de oportunidad positivos si se quieren incentivar conductas, algo similar a los subsidios en cuanto a los precios en la economía (Morales, 2011: 59). Igualmente, al momento de desincentivar una conducta bastará una sanción (gradualmente alta) para tratar de reducirla o eliminarla en la sociedad (Morales, 2011, pág. 59). Para Morales (2011):

El individuo considera entonces la norma como un precio que pondera en sus cálculos junto con otros precios que asigna a sus acciones, y deriva de ellos la utilidad total esperada de sus acciones. En este sentido, las normas jurídicas inciden en las preferencias de los sujetos: las crean, las suprimen o las reordenan. (pág. 59)

Según lo dicho por Catalina Pérez (2014) "la teoría propone que el incremento de en la sanción para un delito $\mathrm{x}$ tendrá como resultado el decrecimiento en la frecuencia con que se comete una conducta y" (pág. 16). Un ejemplo es la aplicación de la denominada "curva negativa de la demanda" al análisis de la eficiencia de las normas jurídicas (Cossío, 2008: 208), la cual formula que, "manteniendo otras variables constantes, un incremento en el precio relativo de cualquier bien disminuirá la cantidad consumida de ese bien... (y que) las variaciones en el ingreso tendrán un efecto de cambiar los gastos totales en igual magnitud (Becker, 1977)" (Cossío, 2008: 208). Siguiendo a Pérez (2014):

Otras versiones de esta teoría afirman que, junto con la severidad de la sanción, lo relevante es la probabilidad de ser sancionado". [...] "En este sentido, para lograr una reducción de la frecuencia con la que se realiza una conducta $\mathrm{x}$, se debe no solo establecer una sanción que en costo supere la ganancia estimada para quien lleva a cabo la conducta delictiva, sino asegurar que dicha sanción sea efectivamente aplicada (Freedman, 2008).

Otros estudios, realizados desde la misma postura, sostienen que el desincentivo tiene mayor relación con la percepción de ser aprehendido que con las probabilidades de esta. Lo relevante de una medida, entonces, no es la probabilidad de ser sancionado, sino que se perciba que se va a ser sancionado (pág. 17).

\section{Notas conclusivas}

Una de las consecuencias que cabe plantear en este trabajo es la necesidad de dotar al derecho de un discurso que permita explicar, de forma más práctica, su objeto en la sociedad. Si a lo largo de la historia, el derecho ha impuesto deberes y obligaciones, y el propio estado de derecho se ha impuesto como un límite al poderoso, ¿cómo estructurar un sistema de organización social que imponga restricciones, deberes y obligaciones a las instituciones que por definición deben buscar la maximización de sus beneficios, como son las empresas?

Los sujetos de derechos claves en el marco de la globalización son las empresas y las preguntas y respuestas que se den en torno a estas definirán tanto los parámetros de investigación como los de enseñanza y de organización social en una sociedad. A este respecto considero que resulta asombrosamente útil el Análisis Económico del Derecho, que abandona grandes y antiguos dogmas al interior del derecho, planteando una renovada mirada a un fenómeno tan complejo como la regulación de la vida en la sociedad contemporánea. En este sentido, es entendible que las categorías de estudio del derecho sean ahora las de 
la economía, toda vez que es el ámbito social que determina la organización de la vida en sociedad, desde una perspectiva global, de ahí tanto interés por parte de diferentes instituciones a nivel mundial y nacional de unificar de alguna forma los sistemas jurídicos (Rodríguez, 2008: 7 - 10). Desde esta perspectiva, las normas jurídicas deben plantearse como formas de resolver problemas, no de provocarlos (como muchas veces pasaba en una visión moderna del derecho), de ahí la proliferación de formas más económicas de resolución de conflictos y producción normativa. Al respecto dice Teubner (2010):

En el proceso de creación del derecho la atención se desplaza ahora hacia los regímenes privados, esto es, hacia los acuerdos obligatorios entre los actores globales, la regulación del mercado privado mediante empresas multinacionales, las reglamentaciones internas en las organizaciones internacionales, los sistemas de negociación interorganizativos $\mathrm{y}$ los procesos mundiales estandarizado. (pág. 72).

Por lo tanto, estandarizar el estado de derecho, su vivencia, estudio e investigación, cumple con los fines de la globalización (Rodríguez, 2008: 17). El análisis económico del derecho juega un papel fundamental en esta estandarización, toda vez que el liberalismo económico de corte neoclásico es la teoría imperante en la economía mundial y la causa de la globalización, sus categorías teóricas sirven como fuente principal para el estudio diversos campos sociales como el derecho, con herramientas más eficaces y menos teóricas puras, que den soluciones rápidas y oportunas a los problemas sociales. Hacia este mismo horizonte apunta el convencionalismo jurídico, que desde la tradición positivista clásica del derecho, permite aportar una explicación que sirve para entender el origen y funcionamiento de los sistemas jurídicos de todos los Estados y sujetos de derecho, a partir de la toma en consideración de las conductas sociales como regulares, obligatorias y arbitrarias, explicación que no riñe con la visión de las normas jurídicas como precios, sino que por el contrario, se encuentran en una misma posición. Desde esta perspectiva, el Derecho no es más que es una convención, que existe de forma parecida al dinero, según Vilajosana (2010):
En este sentido, al igual que sucede con el dinero, sólo podrá ser considerado Derecho de una determinada sociedad lo que ciertos agentes identifiquen como Derecho de esa sociedad” (...) "Del mismo modo que determinados trozos de papel pueden dejar de ser dinero en una sociedad en el momento en que sus usuarios dejen de creer que lo son y actúen en consecuencia (dejen de otorgarles valor y no los acepten como instrumento de intercambio, por ejemplo debido a un fenómeno de hiperinflación) y ello aunque los haya puesto en circulación el organismo competente y los avale el Estado, igualmente unas normas o el sistema jurídico en su conjunto pueden dejar de ser Derecho en una determinada comunidad si los juristas en general dejan de creer que lo son y actúan en consecuencia. (Pág. 160-161)

\section{Referencias bibliográficas}

Arena, F. (2014). El convencionalismo jurídico. Un recorrido analitico. Marcial Pons. Madrid.

Arena,F. (2012). Desacuerdos ambiguos, convenciones equívocas. En Luque, P, y Ratti, G (eds.). (2012). Acordes y desacuerdos. Cómo y por qué los juristas discrepan. Marcial Pons. Madrid.

Cossío, J. (2008). Derecho y Análisis económico. Fondo de Cultura Económica / Instituto Tecnológico Autónomo de México. México D.F.

Hart, H.L.A. (1963). El concepto de Derecho. Abeledo-Perrot. Buenos Aires.

Mercado, D. (2008). Manual de Teoría Constitucional. Rodríguez Quito Editores. Bogotá.

Morales, C. (2011). Las bases del análisis económico del derecho. En Morales (et ál.) Análisis económico del derecho. Siglo del Hombre Editores / Universidad de los Andes / Pontificia Universidad Javeriana - Instituto Pensar. Bogotá.

Moreso, J, Vilajosana, J. (2004). Introducción a la teoría del Derecho. Marcial Pons. Madrid. 
Narváez, M. (2004). Wittgenstein y la teoría del Derecho. Una senda para el convencionalismo jurídico. Marcial Pons. Madrid.

Papayannis, D, Ramírez, L. (2012). Una respuesta pluralista al problema de los desacuerdos jurídicos. En Luque, P, y Ratti, G (eds.). (2012). Acordes y desacuerdos. Cómo y por qué los juristas discrepan. Marcial Pons. Madrid.

Pérez, C. (2014). Por qué las personas obedecen al derecho: desincentivo, normativismo y cumplimiento del derecho. En Tyler, T. $L a$ obediencia del derecho. Siglo del Hombre Editores / Universidad de los Andes / Pontificia Universidad Javeriana - Instituto Pensar. Bogotá.

Rodríguez, C. (2009). La globalización del estado de derecho: el neoconstitucionalismo, el neoliberalismo y la transformación institucional en América Latina. Universidad de los Andes. Bogotá.
Teubner, G. (2010). Regímenes globales privados: ¿derecho neoespontáneo y constitución dual de sectores autónomos? En Saldivia, L. (Comp.). Estado, Soberanía y Globalización. Siglo del Hombre Editores / Universidad de los Andes / Pontificia Universidad Javeriana - Instituto Pensar. Bogotá.

Vilajosana, J. (2010) El Derecho en acción. La dimensión social de las normas jurídicas. Marcial Pons. Madrid.

Weber, M. (2012). Economía y Sociedad. Esbozo de sociología comprensiva. Fondo de Cultura Económica. México D.F.

Viehweg, T. (1997). Tópica y filosofía del Derecho. Gedisa. Barcelona. 